\title{
Photolatent Catalysts
}

\author{
Kurt Dietliker*, Jean-Luc Birbaum, Rinaldo Hüsler, Gisèle Baudin, and Jean-Pierre Wolf
}

\begin{abstract}
Although radiation curing is an established technology used in many industrial manufacturing processes, new applications and technical challenges stimulate the development of tailor-made photolatent catalysts. The development of a new bisacylphosphine oxide (BAPO) photoinitiator enables the efficient curing of coatings stabilized with a light stabilizer package consisting of a UV-absorber and a hindered amine light stabilizer (weather-resistant coatings for outdoor applications). Tailor-made photolatent catalysts represent a key feature in the development of new electronic applications like laser direct imaging for the production of printed circuit boards, color filters for liquid crystal displays used in computer flat screens and chemically amplified photoresists for microelectronics.
\end{abstract}

Keywords: Electronic application · Photo acid generator · Photoinitiator · Photolatent catalyst · Radiation curing

\section{Introduction}

Over the years, radiation curing has found an increasing number of industrial applications and will find use in even more areas in the years to come. The major reasons for this increase are its unique features, including solvent-free formulations, high cure speed and low temperature processing. In addition to these benefits, additional properties of UV curing are of interest today, especially in the automotive industry and in electronic applications.

A key to the success of radiation curing are photoinitiators that meet the requirements of different applications. A variety of compounds are commercially available and find widespread use. Typical examples are suitably substituted acetophenone derivatives that undergo an efficient Norrish type I cleavage upon irradiation. Compounds such as the benzil dimethylketal $\underline{1}$ or the $\alpha$-hydroxy and
${ }^{\text {*Correspondence: }}$ Dr. K. Dietliker

Ciba Specialty Chemicals Inc.

Coating Effects Research

R-1059.5.12

Postfach

$\mathrm{CH}-4002$ Basel

Tel.: + 41616362199

Fax: + 41616362115

E-Mail: kurt.dietliker@cibasc.com $\alpha$-amino ketones $\underline{\mathbf{2}}-\mathbf{6}$ shown in Fig. 1 are the current state-of-the-art for use in many applications including coatings, printing inks or resists. The chemistry and applications of these compounds have been extensively reported elsewhere [1][2].

Novel applications require the development of new photoinitiators with tailor-made properties. Typical examples are the request for photoinitiators which can be used for the curing of stabilized coatings for extended outdoor use, or the requirements in resist technology, which is predominantly driven by the demand for ultra-high resolution required for advanced memory chips and microprocessors. The present paper shows the most important new classes of photolatent catalysts which have been developed to meet these requirements.

\section{Bisacylphosphine Oxide Photoinitiators}

\subsection{Introduction}

Monoacylphosphine oxides (MAPO) have been known as photoinitiators for more than 20 years [3]. The unique properties of this class of photoinitiators were, however, only fully exploited more recently, when furniture manufacturers started to work on UV curable lacquers for white wood coatings.

Continuing research aimed at developing even more efficient photoinitiators for this application led to the recent introduction of bisacylphosphine oxide (BAPO) compounds. Over the last years, this new class of photoinitiators had a significant impact on the development of radiation curing in new applications beyond white

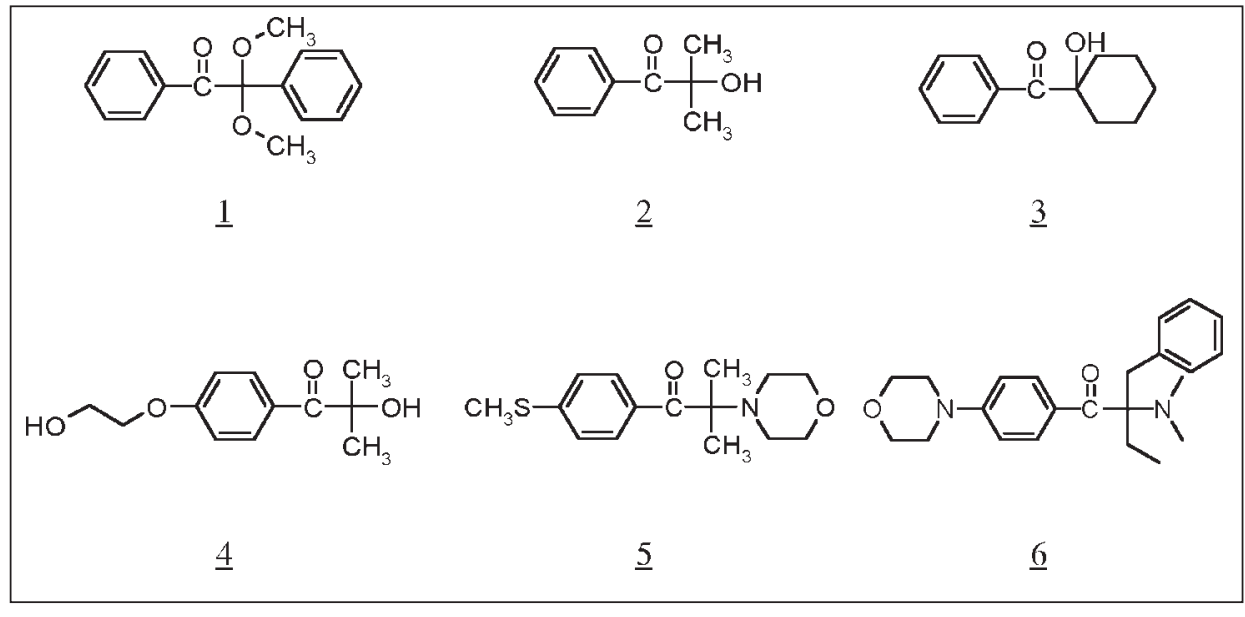

Fig. 1. Typical structures of commercial photoinitiators. 
coatings. The success of these initiators is due to outstanding features that are not matched by other photoinitiators [4-7]. Bis(2,6-dimethoxybenzoyl)-2,4,4-trimethylpentyl-phosphine oxide (플 has been available as part of a photoinitiator blend for several years [8][9]. Further optimization led to the recent introduction of the more efficient new BAPO photoinitiator bis(2,4,6-trimethylbenzoyl)-phenylphosphine oxide (ㅁ) [10] (Fig. 2).

\subsection{Photochemistry}

BAPO photoinitiators have absorption bands in the near UV, which extend well into the visible region. These longwavelength absorptions undergo photobleaching, a property that makes this class of photoinitiators unique. The bleaching process prevents yellowing that is imparted by the photoinitiator and can thus be used $e . g$. in white coatings or clear lacquers despite of the long wavelength absorption.

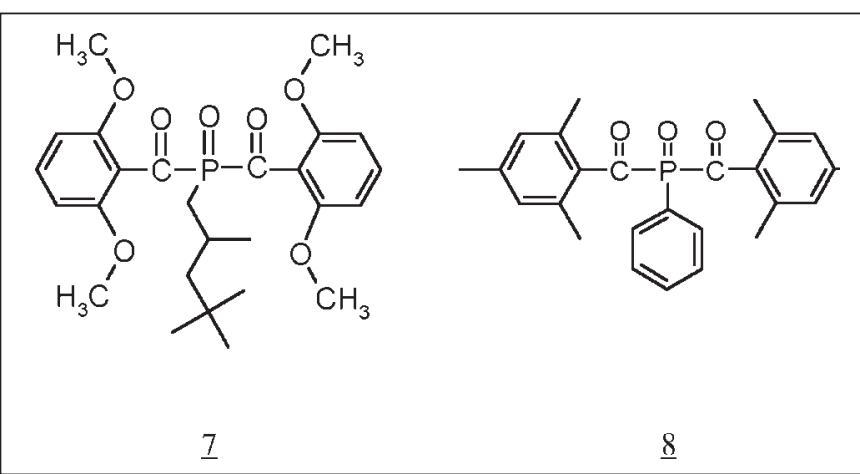

Fig. 2. Bisacylphosphine oxide (BAPO) photoinitiators.

Bleaching also results in a decreasing optical density during irradiation. This allows the incident radiation to penetrate continuously deeper into the coating, thus allowing the curing of thick films. The possibility to produce four initiating radicals in a stepwise manner, compared to two radicals obtained from most other photoinitiators, is another unique feature of BAPO photoinitiators. The stepwise initiation process of bis(2,4,6-trimethylbenzoyl)-phenylphosphine oxide $(\underline{\mathbf{8}})$ is shown in Scheme 1.

These properties translate into a high efficiency under difficult curing conditions such as low light intensity. Bisacylphosphine oxides have thus become the state-of-the-art photoinitiators for applications such as the curing of thick films containing a high loading of white or
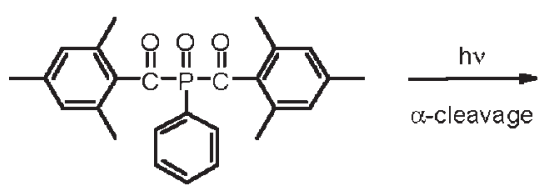

BAPO chromophore absorbing in the visible

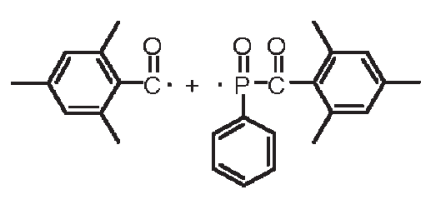

$\stackrel{\mathrm{H}_{2} \mathrm{C} \rightleftharpoons \mathrm{COOCH}_{3}}{\longrightarrow}$

polymer with reactive chain end MAPO chromophore absorbing in the visible
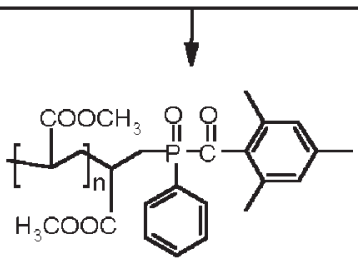

highly reactive benzoyl and phosphinoyl radicals

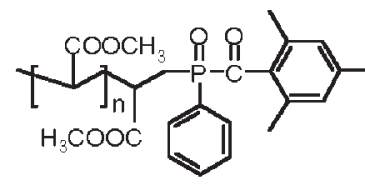

MAPO chromophore absorbing in the visible

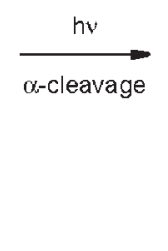

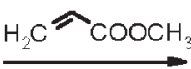
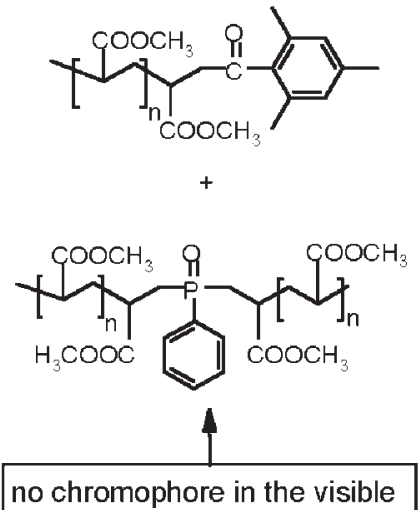

Scheme 1. Stepwise initiation of methyl acrylate polymerisation by bis(2,4,6-trimethylbenzoyl)-phenylphosphine oxide $\underline{8}$. 
colored pigments, as well as for glass fiber reinforced materials or coatings containing other fillers [9][11]. In addition, the availability of these photoinitiators stimulated the development of newly emerging applications, which were not feasible with the photoinitiators available so far.

\subsection{Stabilized UV-Curable Clear Coats for Outdoor Applications}

Coatings for outdoor applications are subjected to harsh weathering conditions by atmospheric influences, such as UV light, oxygen, moisture and air pollutants over a long period of time. This leads to the degradation of the polymeric binder, resulting in effects such as cracking, chalking, color changes, blistering and delamination. Stabilization of outdoor coatings is therefore a must in order to achieve a useful service time of the coating.

The combination of a hindered amine light stabilizer (HALS, e.g. compounds $\underline{9}$ and $\mathbf{1 0}$ in Fig. 3) and a UV-absorber (e.g. compounds $\underline{11}$ or $\underline{\mathbf{1 2}}$ ) is the state-of-theart stabilizer package for outdoor coatings [12]. HALS compounds protect the coating against loss of gloss and cracking. They do not absorb the UV light, but trap any radicals formed during polymer degradation and oxidation. Since highly reactive $\mathrm{N}$-oxyl radicals are only formed in a reaction cycle involving oxygen, HALS stabilizers do not significantly interfere with the curing process.

UV-absorbers filter out the harmful light in the wavelength range of 290-380 nm, thereby protecting the coating against color changes and photochemical degradation that leads to delamination. Efficient UV-absorbers possess absorption maxima with $\varepsilon$ values in the range of $30000-600001 \cdot \mathrm{mol}^{-1} \mathrm{~cm}^{-1}$ in the near UV. This allows the efficient $a b-$ sorption of UV light, which is trans- formed into harmless thermal energy via several deactivation processes [13]. Hydroxyphenyl-benzotriazoles (BTZ) and hydroxyphenyl-s-triazines (HPT) are the most efficient classes of UV-absorbers and find widespread use.

In UV-curable formulations, the UVabsorbers efficiently compete with the photoinitiator for the light in the UV-A region. A comparison of typical absorption spectra of a BTZ and a HPT UV absorber with that of an $\alpha$-hydroxyketone photoinitiator and BAPO/ $\alpha$-hydroxyketone photoinitiator blend is shown in Fig. 4. The concentration ratio of UV-absorber and photoinitiator corresponds to that used in a typical UV-curable coating.

The absorption spectrum of the $\alpha$ hydroxyketone photoinitiator, typically used in clear coatings for indoor applications, is completely masked by the much stronger absorption of the UV-absorber.
The long wavelength absorption of the BAPO photoinitiator, however, extends well into the visible and thus into a region not covered by the UV-absorber. Therefore the BAPO photoinitiator is reactive even in the presence of the UV-absorber. Photobleaching of the initiator is essential, since yellowing is not acceptable in clear coats. Thus, BAPO compounds are the photoinitiators of choice for the curing of stabilized clear coats [14-17].

Essential for the success of UV-cured outdoor coatings is the careful choice and optimum match of photoinitiators and UV-absorbers. Extended studies revealed that BAPO compound $\underline{\mathbf{8}}$ in combination with an $\alpha$-hydroxyketone photoinitiator gives the best overall curing performance. An $\alpha$-hydroxyketone photoinitiator is advantageously added for a very fast curing of the coating surface, where the absorbance of light by the UV-absorber is

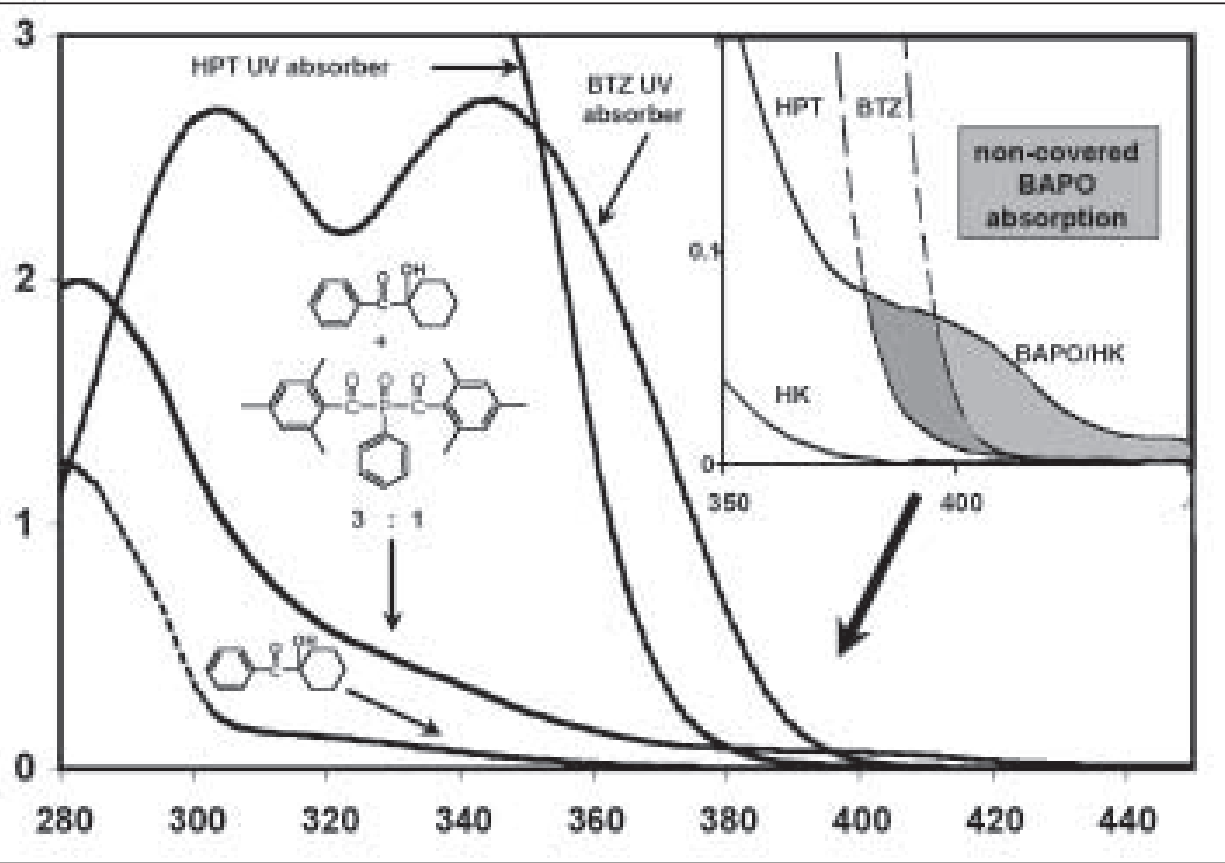

Fig. 4. Absorption spectra of UV-absorbers and photoinitiators. Inset: Absorption of the BAPO photoinitiator $\underline{8}$ not covered by the UV-absorbers. Concentration: $0.02 \mathrm{~g} / \mathrm{l}$.

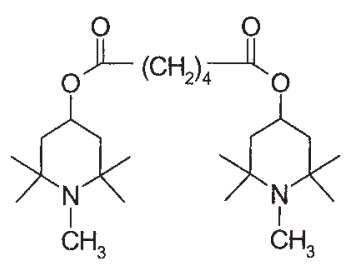
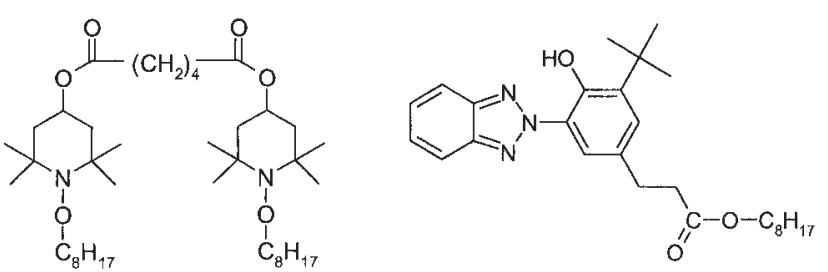

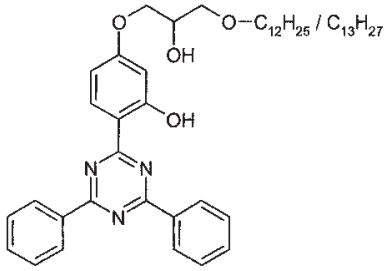

11

Fig. 3. UV-absorbers and HALS stabilizers used in stabilized UV curable clearcoats; $\underline{9}$ : $N$-alkyl HALS, 10: $N$-alkoxy HALS, 11: hydroxyphenylbenzotriazole (BTZ) type UV-absorber, 12: hydroxyphenyl-s-triazine (HPT) type UV-absorber. 
low. HPT-type UV-absorbers are better suited for UV curable coatings than BTZ compounds, since the steeper slope of the absorption band makes more light available for the photoinitiator. However, the choice of the UV-absorber is in the first place made in view of the substrate to be protected. BTZ-type UV-absorbers give excellent results in clear wood coatings, since the red-shifted absorption efficiently absorbs the light harmful to lignin. The protection of pigments against color fading by a clear topcoat in automotive applications is best achieved with an HPT-type UV-absorber. BAPO/ $\alpha$-hydroxyacetophenone combinations are able to cope with both situations.

Aliphatic urethane acrylates are the preferred binders for outdoor coatings, since they provide good mechanical properties combined with high flexibility and excellent yellowing resistance. UV cured coatings of this type have a performance which matches or exceeds that of a thermally crosslinked, two-pack acrylic/NCO resin, which is considered state-of-the-art for these applications [18-21]. The outstanding performance of UV cured clear coats was recently confirmed by the acceptance of this technology for automotive protective coatings, one of the most demanding outdoor applications.

\section{New Photolatent Catalysts for Electronic Applications}

\subsection{Introduction}

Photoresists (radiation-sensitive materials whose solubility is altered when exposed to light) play a crucial role in the production of all electronic appliances [22]. The selective exposure of photoresists (for example, through a mask), followed by suitable physical/chemical treatments, is the key step ('imaging') creating the fine patterns found in every printed circuit board (PCB) or microelectronic device.

The technology is driven by the demand for more reactive materials and higher resolution (smaller feature dimensions). Therefore, the development of new, high-performance photoinitiators remains a major goal for industrial research in that field. Here is just a selection of Ciba's recent achievements.

\subsection{Titanocene Photoinitiators for Laser Direct Imaging}

Laser direct imaging (LDI) is a maskless technique in which the photoresist is directly exposed to the light of a scanning laser beam. It offers a number of advantages in the manufacturing of PCB: no mask registration, dimensional stability, suitability for prototyping and small series, and no yield loss due to mask defects. Since visible-light laser sources are still the most reliable tools, it is critical to have a photoinitiator of high sensitivity in the visible range (Fig. 5). Titanocene derivatives such as $\underline{\mathbf{1 3}}$ and $\underline{\mathbf{1 4}}$ have been shown to be remarkably well suited for these applications [23]. The fluorine substituents are critical for thermal and hydrolytic stability.

The initiation mechanism (Scheme 2) has been partly elucidated for the closely related bis(pentafluorophenyl)titanocene (BPPT) [24].

The primary photo process generates [a], a highly reactive isomer of BPPT, via a cyclopentadienyl ring slippage reaction with unity quantum yield. Then, this very electrophilic and coordinatively unsaturated species reacts with any donor available, for example, an acrylate. From this point on, the mechanism is more speculative. Ketene acetal radicals of structure [d] are the proposed polymerization initiators, but the exact nature of the other ligands at the titanium in [b] and [d] is unknown. The postulated allylic radical [c] is the common intermediate of five isolated decomposition products, acting apparently as a radical quencher, not as initiating species (see termination step in Scheme 2). Increased yields of polymer
Fig. 5. Absorption spectrum of titanocene $\mathbf{1 3}$ $(0.01 \mathrm{~g} / \mathrm{l}$ in acetonitrile), showing good overlap with the emission bands of an argon laser at 488 and $514 \mathrm{~nm}$.

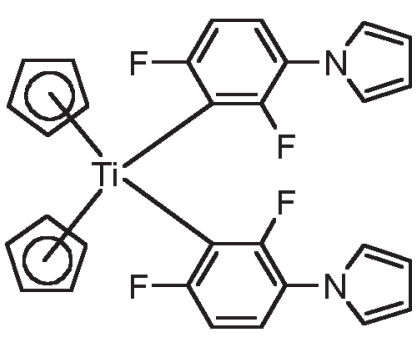

13

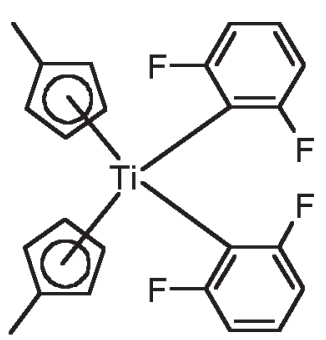

14

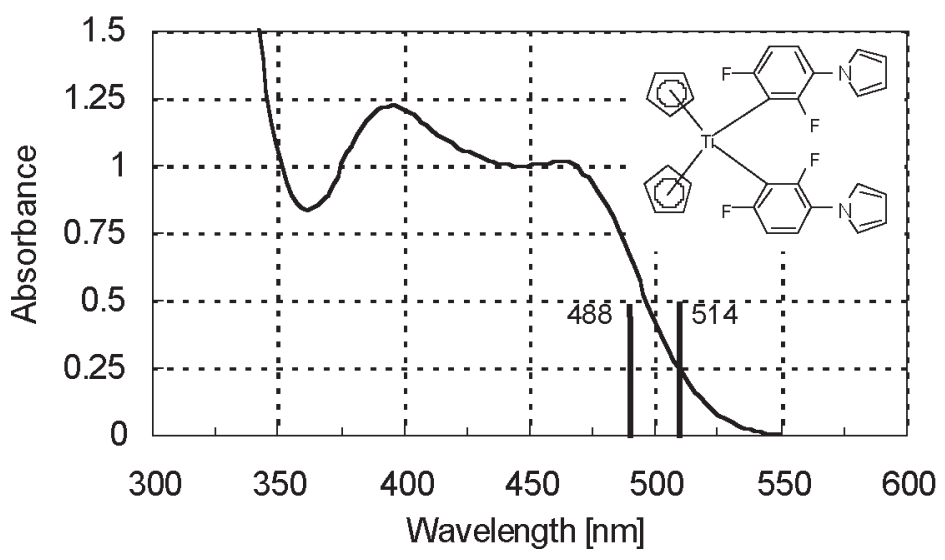




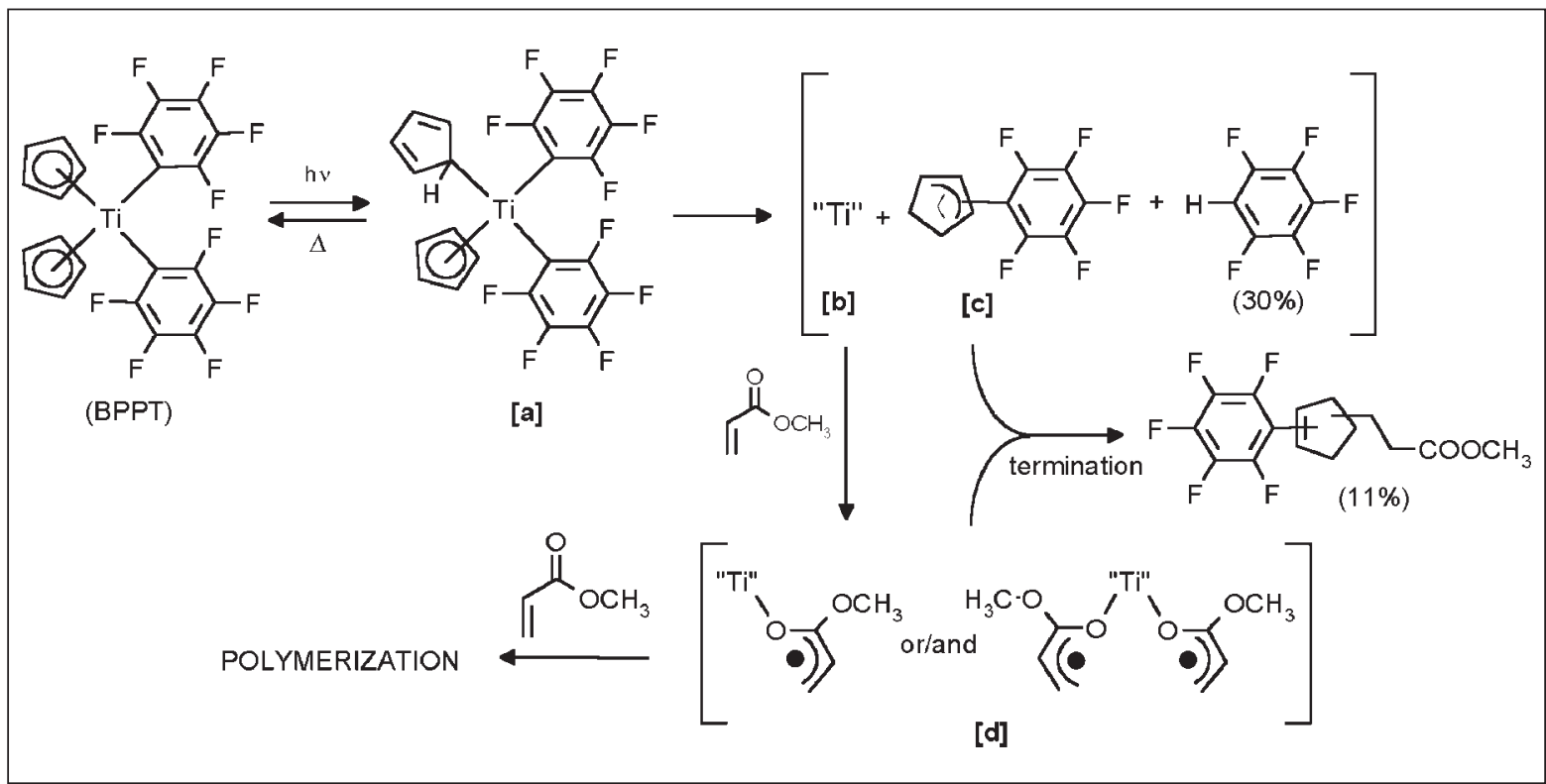

Scheme 2. Proposed initiation mechanism for BPPT. The formation of minor secondary products is not shown. Yields are based on starting BPPT.

observed by adding small amounts of a diketone suggest a chelating effect and support the proposed structure [d] in which one electron is transferred from the titanium to a complexed carbonyl group.

\subsection{Oxime Ester for Color Filters}

The manufacturing of color liquid crystal displays (LCD, used for example in flat computer screens) requires color filter resists of high reactivity and precisely defined transmission spectrum, so that the purity of the fundamental colors is guaranteed. A suitable radical photoinitiator for this application should have a high reactivity, low oxygen sensitivity, and should not cause yellowing of the photoresist upon exposure and thermal treatment. We have found that some red-shifted oxime esters like $\underline{\mathbf{1 5}}$ [25] (Scheme 3) are especially suitable for this application and show advantages over currently used photoinitiators such as trihalomethyltriazines or $\alpha$-aminoketones. The initiation mechanism of oxime esters, which involves primary cleavage of the $\mathrm{N}-\mathrm{O}$ bond and secondary decarboxylation, has been described by Hageman [26].

\subsection{Photo Acid Generators}

The discovery of photolatent acids (compounds which efficiently generate acid when irradiated by UV or visible light) has opened the field of photoinitiated cationic polymerization and polycondensation. Major applications include coatings, adhesives, and inks. Photoresists for microelectronics also represent

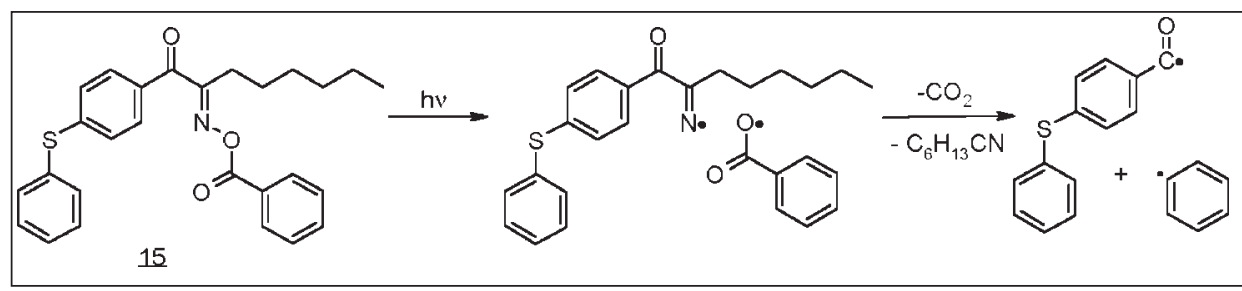

Scheme 3. Photo-induced cleavage mechanism of an oxime ester.

a very important application. In that case, the generated acid may not only initiate cationic polymerization, but can also catalyze depolymerization reactions or catalytically remove protective groups of a functionalized polymer ("chemically amplified photoresists').

Compounds $\underline{16}-\underline{18}$ (Fig. 6) are representative examples of cationic photoinitiators developed at Ciba. The iron(II) arene complex $\underline{\mathbf{1 6}}$ has been found to be highly suitable for imaging applications using solid, multifunctional novolak epoxy compounds [27]. Its initiation mechanism, based on photoinduced areneepoxy ligand exchange and epoxy ring opening, has been described elsewhere [28]. The iodonium salt $\underline{\mathbf{1 7}}$ is a valuable cationic initiator for highly pigmented white base container coatings and flexographic inks because it does not contain heavy metals and responds extremely well to photosensitizers such as thioxanthones [29].

The oxime sulfonate $\underline{\mathbf{1 8}}$ is a good example of a non-ionic and halogen-free photolatent acid which gives superior performance in chemically amplified pho- toresists under irradiation conditions ranging from the deep-UV (248 nm, $\mathrm{KrF}$ laser) to the g-line of mercury lamps $(436 \mathrm{~nm})$ [30].

\section{Conclusion}

In modern coatings and photoresists, photoinitiators are much more than simple polymerization starters: they are an integral part of sophisticated compositions that must fulfill high technical and esthetic requirements such as light fastness, durability, and protection. Curing is only the first step in the lifetime of a coating or a photoresist. Therefore, the design of photoinitiators must include the conditions imposed by the other components of the formulation. For example, in coatings, BAPO overcome the problem of competitive light absorption by pigments or UV-absorbers; in the case of LCD, oxime esters improve the transmission spectrum of the finished color filters. These tailor-made properties add real value to photoinitiators and open new applications for radiation curing. 


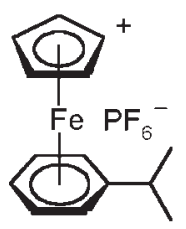

16

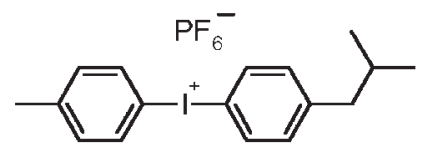

17

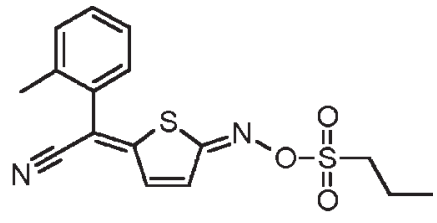

18

Fig. 6. Examples of photo acid generators.

Further, photoinitiator research is currently aimed at the development of novel types of photolatent catalysts, such as photobase generators [2]. These catalysts will allow radiation curing to penetrate into new types of resin chemistry, which provide advantages in different types of applications. First examples are currently under development [31] and industrial applications are expected to be on the market in the near future. Hence research is constantly striving for technological innovations that further broaden the scope of radiation curing.

Received: March 28, 2002

[1] A.F. Cunningham, V. Desobry, K. Dietliker, R. Hüsler, D.G. Leppard, Chimia 1994, $48,423$.

[2] K. Dietliker, in 'Photoinitiators for Free Radical Cationic and Anionic Photopolymerisation', vol. III in the series 'Chemistry and Technology of UV and EB Formulation for Coatings, Inks and Paints'; John Wiley and Sons/SITA Technology Limited, London, 1998.

[3] M. Jacobi, A. Henne, J. Radiation Curing 1983, 10(4), 16.

[4] U. Kolczak, G. Rist, K. Dietliker, J. Wirz, J. Am. Chem. Soc. 1996, 118, 6477.

[5] S. Jockusch, N.J. Turro, J. Am. Chem. Soc. 1998, 120, 11773.

[6] I. Gatlik, P. Rzadek, G. Gescheidt, G. Rist, B. Hellrung, J. Wirz, K. Dietliker, G. Hug, M. Kunz, J.-P. Wolf, J. Am. Chem. Soc. 1999, 121, 8332.

[7] R.M. Williams, I.V. Khudyakov, M.B. Purvis, B.J. Overton, N.J. Turro, J. Phys. Chem. B 2000, 104, 10437.

[8] D. Leppard, K. Dietliker, G. Hug, R. Käser, M. Köhler, U. Kolczak, L. Misev, G. Rist, W. Rutsch, Proc. Radtech'94 North America, vol. II; Northbrook, 1994, p. 693.

[9] W. Rutsch, K. Dietliker, D. Leppard, M. Köhler, L. Misev, U. Kolczak, G. Rist, Prog. Org. Coatings 1996, 27, 227.

[10] K. Dietliker, D. Leppard, T. Jung, M. Köhler, A. Valet, U. Kolczak, P. Rzadek, G. Rist, Conf. Proc. RadTech Asia 97, Yokohama, 1997, p. 292.

[11] W. Rutsch, H. Angerer, V. Desobry, K. Dietliker, R. Hüsler, Proceedings of the 16th International Conference in Organic Coatings Science and Technology, July 9-13, 1990, Athens, Greece, p.423.
[12] A. Valet, Farbe Lack 1990, 96, 189.

[13] H.E.A. Kramer, Farbe Lack 1985, 92, 919.

[14] C. Decker, Proc. XXIth International Conference in Organic Coatings Science and Technology, Athens, 1995, p. 153.

[15] A. Valet, T. Jung, M. Köhler, Farbe Lack 1998, 104(2), 42.

[16] A. Valet, D. Wostratzky, Proc. RadTech'98 North America, Chicago, 1998, p. 396.

[17] A. Valet, Prog. Org. Coat. 1999, 35, 223.

[18] C. Decker, K. Zahouily, A. Valet, Polym. Mater. Sci. Eng. 2000, 83, 323.

[19] S. Megert, Polym. Paint Colour J. 2000, 190(4432), 9.

[20] A. Valet, C. Decker, Mod. Paint Coat. 2001, 91(5), 29.

[21] T. Jung, A.Valet, RadTech Report 2001 (Nov/Dec), 30.

[22] H. Steppan, G. Buhr, H. Vollmann, Angew. Chem. 1982, 94, 471-485.

[23] T. Doba, RadTech Asia '93 UV/EB Conf. Exhib, Conf. Proc. 1993, 193-7. RadTech Japan, Tokyo.

[24] a) B. Klingert, A. Roloff, B. Urwyler, J. Wirz, Helv. Chim. Acta 1988, 71, 1858; b) J. Finter, M. Riediker, O. Rohde, B. Rotzinger, Makromol. Chem. Makromol. Symp. 1989, 24, 177.

[25] a) M. Kunz, H. Oka, H. Kura, RadTech 2000 (USA) Conf. Proc. 2000, 346-357; b) H. Kura, H. Oka, K. Kunimoto, J. Tanabe, M. Ohwa, International Display Workshop '00, Proc. 2000, 355-358.

[26] C.G. Groenenboom, H.J. Hageman, P. Oosterhoff, T. Overeem, J. Verbeek, $J$. Photochem. Photobiol. A 1997, 107, 261; H.J. Hageman, P. Oosterhoff, J. Verbeek, J. Photochem. Photobiol. A 1999, 121, 207.

[27] K. Meier, H. Zweifel, J. Imaging Sci. 1986, 30, 174

[28] K. Meier, G. Rihs, Angew. Chem. 1985, 97, 879; K. Meier, Coord. Chem. Rev. 1991, 111, 97.

[29] J.-L. Birbaum, S. Ilg, RadTech Europe 2001, Conf. Exhib. Basel, Oct. 8-10, 2001, Conf. Proc. p. 545-550.

[30] T. Asakura, H. Yamato, A. Matsumoto, M. Ohwa, Proc. SPIE-The International Society for Optical Engineering 2001, 4345 (Pt.1, Advances in Resist Technology and Processing XVIII), 484-493.

[31] S. Turner, G. Baudin, WO 9841524 A1 (Prior. 18.3.1997) to Ciba Specialty Chemicals Inc. 